\title{
A MICRO-RESONATOR BASED DIGITAL TO ANALOG CONVERTER FOR ULTRALOW POWER APPLICATIONS
}

\author{
Sally Ahmed, Xuecui Zou, and Hossein Fariborzi*. \\ Computer, Electrical, and Mathematical Science and Engineering Division (CEMSE), King \\ Abdullah University of Science and Technology (KAUST), Saudi Arabia.
}

\begin{abstract}
This paper presents a micro-resonator based digital to analog converter (DAC) for the internet of things (IoT) and low power applications. The device consists of a clamped-clamped micro-resonator with multiple electrodes. To enable the operation as a DAC, the air-gap between each partial electrode and the beam is varied based on the weight of input bits, so the beam's resonance frequency and the output amplitude are decided by digital inputs. The tested DAC consumes $7 \mathrm{pJ} /$ conversion step with a sampling rate of $64 \mathrm{~S} / \mathrm{s}$. We show that a sampling rate of $0.1 \mathrm{MS} / \mathrm{s}$ and energy/conversion of $2.7 \mathrm{fJ}$ can be achieved by moderate device scaling.
\end{abstract}

\section{KEYWORDS}

Digital to Analog Converters, Micro-resonators, Electromechanical Logic, Electrostatic Softening Effect.

\section{INTRODUCTION}

Energy efficiency has become one of the most important requirements for the stand-alone batteryoperated IoT devices. In addition, these devices only require moderate processing speeds (sub-kHz to 100 $\mathrm{MHz}$ ) and a form factor of sub-millimeters to tens of centimeters [1]. This makes nano and microelectromechanical (N/MEM) relays and resonators appealing for the IoT applications, as they have been shown to be more energy-efficient than CMOS transistors in many design spaces. Although N/MEM relays have zero leakage current, they suffer from contact resistance and wearing issues [2]. On the other hand, the operation of N/MEM resonators does not involve direct contact. It depends on the vibration state of the resonating part, and hence resonators are more reliable than relays. The applications of N/MEM resonators have been limited to sensors, actuators and oscillators until 2007 when the first micro-resonator based logic gate was proposed [3]. Unlike CMOS, there are no well-defined methods and rules for implementing logic circuits using resonators and researchers have explored many different design techniques. One of these techniques is based on the vibration mode of the resonator, where different modes can be used to implement different logic functions [4-5]. Some other techniques have been used for implementing reprogrammable logic gates, such as shifting the resonance frequency of a resonating beam using DC current flowing through a resonator beam [6], or through the electrostatic softening effect from partial electrodes [7]. Most of the micro-resonator based logic devices presented so far are limited to simple logic gates and memory elements. In order to build functional IoT systems, more complicated blocks are required, such as digital signal processing (DSP) units and data converters.
In a previous work [8], we presented a more complicated digital block, namely a full adder, which can be considered as the basic block of all arithmetic operations and digital signal processing. The proposed micro-resonator adder required 2 resonators, which is $\sim 14$ times less complex than the conventional optimized CMOS adders. Two of the missing components of a micro-resonator based VLSI system are data converters (digital to analog (DAC)/ analog to digital (ADC) converters), which are crucial elements of sensorprocessor interfaces. In this paper, a novel design of a micro-resonator based DAC is presented. The operation of the device is verified by simulation and experiments.

\section{DEVICE STRUCTURE AND OPERATION}

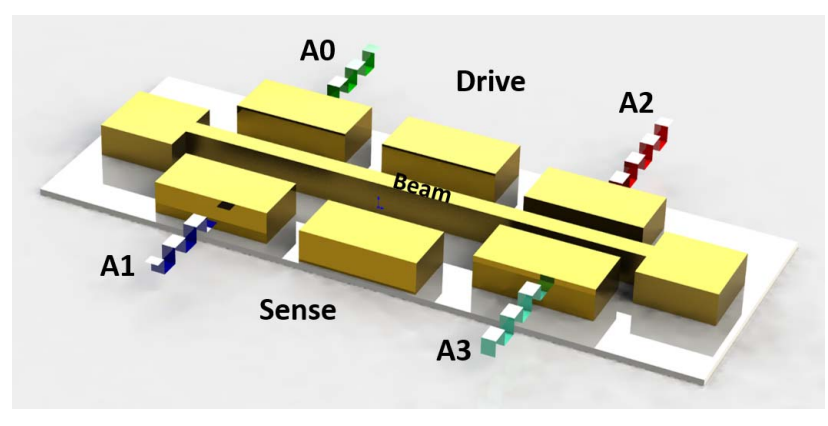

Figure 1: 3D schematic of the proposed micro-resonator based DAC with weight adjusted electrode/beam air gaps.

Figure 1 shows a 3D schematic of the proposed single-device 4-bit micro-resonator DAC which consists of a clamped-clamped silicon beam with multiple side electrodes. One of the middle electrodes is used for electrostatically actuating the beam by applying an $\mathrm{AC}$ signal with the frequency of interest. The output current is detected at the sense electrode on the opposite side of the beam. The digital inputs, which are DC voltages applied to the corner electrodes, modulate the resonance frequency of the beam. For a device with input electrodes separated from the beam by equal air gaps in a previous work [8], simulation and experimental results showed that the combinations that contained the same number of ones/zeros (regardless of their order) had the same effect on the resonance frequency of the beam. For example, the input combinations 0001-0010-0100-1000, which correspond to the decimal equivalent values 1-2-4-8, resulted in almost the same resonance frequency for the beam. For a digital to analog converter, there should be a one to one correspondence between the digital input combinations and the output, unlike the logic gates where multiple input combinations can result in the same output. Therefore, we redesigned the device, such that the more significant bits have a higher impact on the beam's 
resonance frequency. This has been achieved by decreasing the size of the air gap $(\mathrm{g})$ between the beam and the input electrodes from the least significant bit (LSB) to the most significant bit (MSB), such that $\mathrm{g}_{\mathrm{A} 0}>\mathrm{g}_{\mathrm{A} 1}>\mathrm{g}_{\mathrm{A} 2}>\mathrm{g}_{\mathrm{A} 3}$.

\section{SIMULATION AND EXPERIMENTAL RESULTS}

\section{Methodology and Simulation Results}

COMSOL (a finite element software) simulations were performed to determine the required air-gaps that guarantee a constant increase in the resonance frequency of the beam when the digital inputs have higher equivalent decimal values. The selected dimensions of the device are shown in Figure 2. The digital inputs are DC voltages applied to the four corner electrodes, where digital ' 0 ' is $0 \mathrm{~V}$ and digital ' 1 ' is $20 \mathrm{~V}$. The input vector consists of 4 bits $A_{3} A_{2} A_{1} A_{0}$, where $A_{3}$ is the MSB and $A_{0}$ is the LSB. The beam is biased with $40 \mathrm{~V}$. Parametric sweep is performed to obtain the beam's resonance frequency for all sixteen combinations. Device optimization started from a device with equal air gaps between the beam and the side electrodes. As mentioned earlier, in such configuration, the inputs that had the same number of digital ones (zeros) resulted in the same resonance frequency. The optimization starts by reducing the air gap between the electrode of the $\operatorname{MSB}\left(\mathrm{A}_{3}\right)$ and the beam while keeping all the other electrodes at the same distance from the beam. That results in an increased resonance frequency for the last eight combinations in which the MSB is ' 1 '. Next, the air gap between the beam and the electrode of the second significant bit $\left(\mathrm{A}_{2}\right)$ is reduced, while keeping it larger than $\mathrm{g}_{\mathrm{A} 3}$. That results in an increased resonance frequency for the combinations in which $A_{2}$ is ' 1 ', compared to those in which $A_{2}$ is ' 0 '. The same procedure can be repeated for $A_{1}$ and $A_{0}$ until a monotonic increase in the resonance frequency is obtained as the input varies from 0000 to 1111 . Figure 2 shows the resonance frequency for each combination after beam/electrode air gap optimization. Changing the beam bias or the digital ' 1 ' voltage will only change

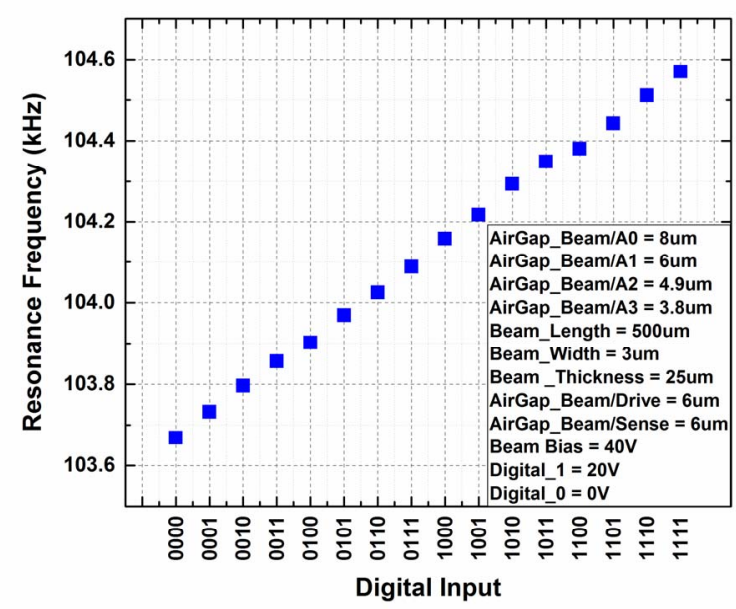

Figure 2: COMSOL Simulations of the proposed device showing the resonance frequency of the beam for each input combination. the frequency separation between the different states, following the same trend. All zeros combination results in the lowest resonance frequency due to the electrostatic softening effect as this case represents the maximum voltage difference between the beam and the input electrodes. For the case of all ones, the voltage difference between the beam and the electrodes is minimum which results in the highest resonance frequency.

\section{Experimental Results}

Experiments were performed on silicon resonators fabricated by MEMSCAP using SOIMUMPs process. Figure 3 shows the experimental setup and a scanning electron microscopy (SEM) image of the fabricated DAC. A network analyzer is used to supply the drive signal and measure the output from the sense electrode after it passes through a low noise amplifier (LNA) with a unity gain. Due to some variations between the designed and fabricated air gaps and also due to possible residual stresses in the beam, that were not accounted for in the simulation, only a 3-bit DAC showed the perfect expected behavior. So, we left the electrode of $\mathrm{A}_{0}$ floating in the experiments. The beam is $500 \mu \mathrm{m}$ long, approximately 3 $\mu \mathrm{m}$ wide and $25 \mu \mathrm{m}$ thick. The sense and drive electrodes have $6 \mu \mathrm{m}$ air gap separation from the beam. The beam is biased at $40 \mathrm{~V}$ and the digital ' 1 ' ' is also $40 \mathrm{~V}$ to ensure the desired separation between the different peaks. The device was tested in a vacuum chamber under 40 mTorr pressure and in room temperature.

Figure 4 shows the experimental results of a 3-bit DAC where the forward and backward frequency sweeps of each combination are plotted. $A_{1}, A_{2}$, and $A_{3}$ are used for applying digital inputs and $\mathrm{A}_{0}$ is left floating. As predicted by simulation, the resonance frequency increases as the equivalent decimal value of the applied digital inputs increases. The input power was chosen such that the bandwidth of the forward sweep of the 000 case is wide enough to overlap with the mono-stable region of the 111 case (the minimum required input power). The frequency of operation is chosen to be at the maximum output of the mono-stable region when 111 is applied. The frequency shift between the different peaks causes the resonator to have different output levels at the frequency of operation shown in Figure 4. For correct operation, the DAC should be initialized with 111 .

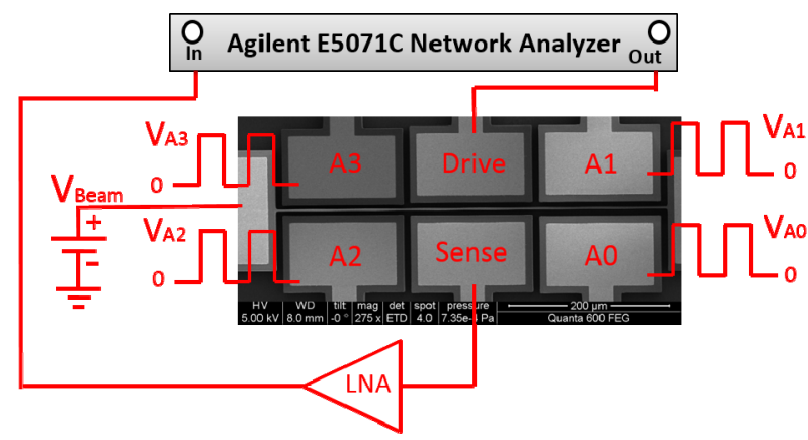

Figure 3: SEM picture of the proposed device, showing the experimental set-up. 


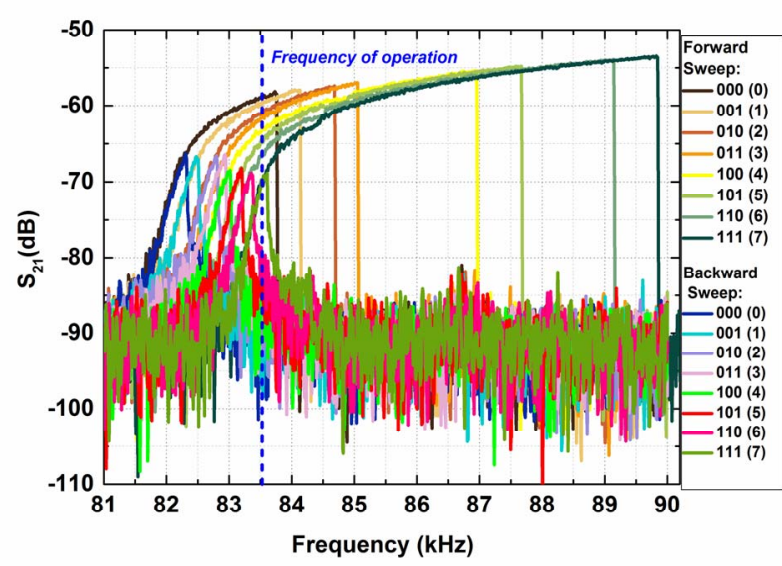

Figure 4: Experimental results showing the frequency response of a 3-bit DAC, forward and backward sweeps are plotted for each combination.

Figure 5 shows the time response of the resonator when the frequency of the drive signal is fixed at 3 different frequencies in the mono-stable region of the 111 case and also for different input powers. The lowest output level corresponds to the 111 case while the highest level corresponds to 000 case. The digital inputs can be inverted to obtain an increasing level from 000 to 111 . As the input power increases, the step size between the different levels becomes smaller, leading to better accuracy and linearity for the DAC.

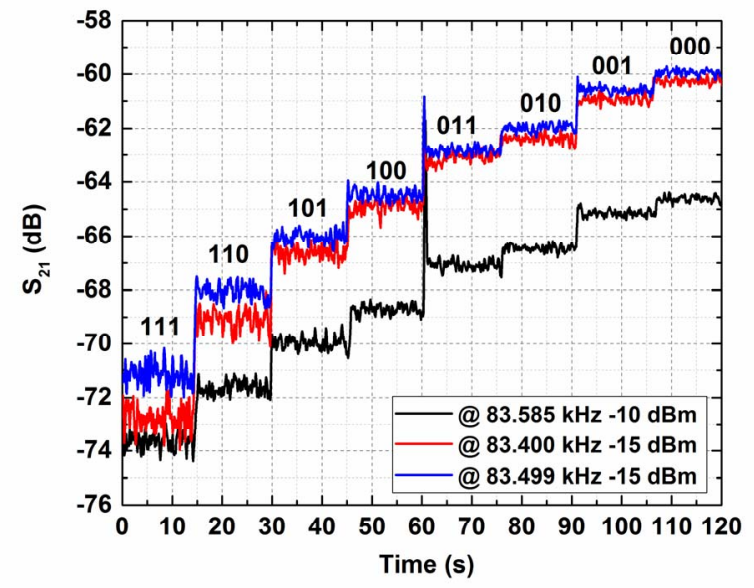

Figure 5: Time response of the DAC at 3 different frequencies in the mono-stable region for 111 case and at 2 different input power levels.

\section{DISCUSSION}

For proper DAC operation, it is important to guarantee successful transitions between any two different levels. By fixing the input power to $-15 \mathrm{dBm}$, and trying to switch between the different cases, we found out that correct switching occurs in the following transitions ( 111 to 110$)$, and (111 to 101). Transitions to higher states were not successful. Therefore, we started to increase the applied power while fixing the frequency of operation to $83.5 \mathrm{kHz}$. We noticed that as the input power increases, more successful transitions to higher levels occur. In addition, we noticed that the difference between the output levels is smaller as shown in Figure 5. This is explained by noting that as the $\mathrm{AC}$ power increases, the bandwidth of the mono-stable response becomes larger and the peak shifts to the right. This results in a lower output at the frequency of operation that was fixed at 83.5 $\mathrm{kHz}$. Currently, more optimizations are carried out to guarantee a successful device operation.

Other important aspects of DAC operation is the sampling speed and energy consumption. The speed of the micro-resonator device is a function of the quality factor $Q$ and the resonance frequency $f$ of the beam. The measured $Q$ of the resonator is around 1300 while operating under the pressure of 40 mTorr. The transition time ( $\left.t_{\text {swtiching }}\right)$ is defined as $Q / f$ [9] which is $15.56 \mathrm{~ms}$, yielding a sampling speed of $64 \mathrm{~S} / \mathrm{s}$. The speed of the operation can be increased significantly by scaling the resonator to achieve higher resonance frequencies and lower quality factor. Figure 6 shows the dimensions and resonance frequency tuning of a moderately scaled resonator working around $9.4 \mathrm{MHz}$ resonance frequency. Assuming $Q$ of 100, the expected sampling rate will be approximately $0.1 \mathrm{MS} / \mathrm{s}$. Further optimizations of the device dimensions and proper material choice can increase the resonance frequency to $\mathrm{GHz}$, which eventually will increase the sampling speed to $\mathrm{MS} / \mathrm{s}$.

The other important aspect is the energy consumption. The energy consumed by this device consists of switching energy and activation energy. By adjusting the equation of switching energy in [8] and taking into account that the capacitances between the beam and the input electrodes are not equal, the average switching energy per operation for the tested 3 bit DAC is $8 \mathrm{pJ}$ which corresponds to $1 \mathrm{pJ}$ energy per conversion step. The estimated AC activation energy per conversion step is around $6 \mathrm{pJ}$. Therefore, the total energy/conv step is $7 \mathrm{pJ}$. An optimized and moderately scaled 4 bit DAC, with specifications shown in Figure 6, consumes 2.7 fJ per conversion step, which is 3 orders of magnitude lower than the energy consumption of the tested device.

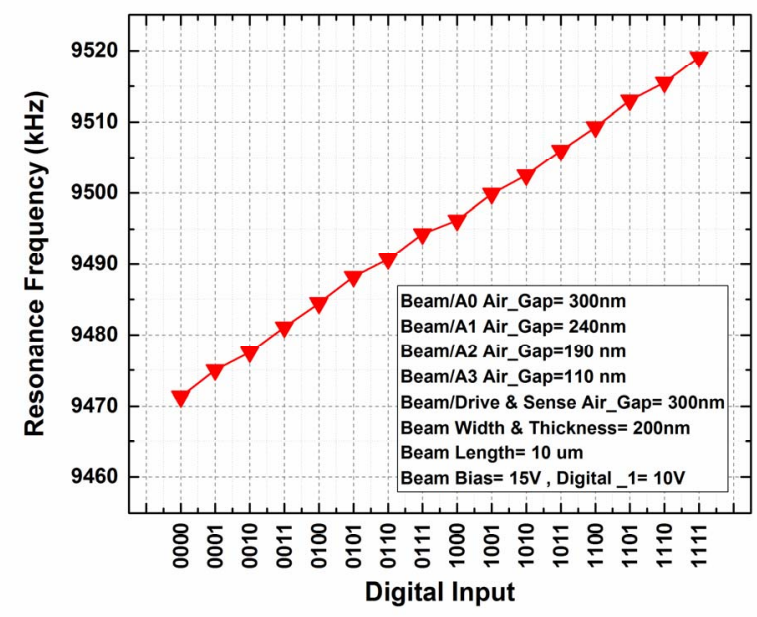

Figure 6: COMSOL simulations for a moderately scaled resonator showing the required air gaps for obtaining a $D A C$. 
Although the tested micro-resonator DAC consumes $7 \mathrm{pJ} /$ conv step, which is 30 to 100 times more than CMOS DACs in $65 \mathrm{~nm}$ and $40 \mathrm{~nm}$ technologies [10-11], the optimized device reduces the energy per conversion step by 3 orders of magnitude, thereby beating its CMOS counterparts. Although the speed of micro-resonator DACs is not comparable with CMOS counterparts, they are still appealing solutions for IoT applications with low or moderate speed requirements [1].

\section{CONCLUSION}

A novel approach for implementation of a low-power digital to analog converter is presented. The entire conversion is performed by a single micro-resonator device. The operation of the proposed device is based on the modulation of resonance characteristics of the microbeam through the applied digital inputs. The weight of each input is adjusted through the air gaps between the input electrodes and the beam. While the fabricated device is relatively slow and consumes a few picojoules per conversion step, proper optimization can increase the sampling speed and further reduce energy consumption to an order of magnitude lower than CMOS DACs.

\section{ACKNOWLEDGMENTS}

The authors would like to thank Nizar Al Jaber, Saad Ilyas and Prof. Mohammad Younis from the PSE division at KAUST for their feedback. This work is supported by King Abdullah University of Science and Technology (KAUST) office of sponsored research (OSR) under Award No. OSR-2016-CRG5-3001.

\section{REFERENCES}

[1] D. Blaauw et al., "IoT design space challenges: Circuits and systems," 2014 Symposium on VLSI Technology (VLSI-Technology): Digest of Technical Papers, Honolulu, HI, 2014, pp. 1-2.

[2] D. A. Czaplewski, C. D. Nordquist, C. W. Dyck, G. A. Patrizi, G. M. Kraus, and W. D. Cowan, "Lifetime limitations of ohmic, contacting RF MEMS switches with $\mathrm{Au}, \mathrm{Pt}$ and Ir contact materials due to accumulation of 'friction polymer'on the contacts." $J$. Micromech. Microeng, vol. 22, pp. 105005, 2012.

[3] S. C. Masmanidis, R. B. Karabalin, I. De Vlaminck, G. Borghs, M. R. Freeman, and M. L. Roukes, "Multifunctional nanomechanical systems via tunably coupled piezoelectric actuation.", Science, vol. 317, pp. 780-783, 2007.

[4] D. Hatanaka, I. Mahboob, H. Okamoto, K. Onomitsu, and H. Yamaguchi, "An electromechanical membrane resonator," Appl. Phys. Lett., vol. 101, p. $063102,2012$.

[5] S. Ilyas, S. Ahmed, M. Hafiz, H. Fariborzi and M. Younis, "Cascadable microelectromechanical resonator logic gate", J. Micromech. Microeng, vol. 29, no. 1, p. 015007, 2018.

[6] M. A. A. Hafiz, L. Kosuru, and M. I. Younis, "Microelectromechanical reprogrammable logic device," Nat. Commun., vol. 7, p. 11137, 2016.

[7] M. A. A. Hafiz, S. Ilyas, S. Ahmed, M. I. Younis, and H. Fariborzi, "A Microbeam Resonator with Partial
Electrodes for Logic and Memory Elements," IEEE J. Explor. Solid-State Computat. Devices Circuits, vol. 3, pp. 83-92, 2017.

[8] S. Ahmed, S. Ilyas, X. Zou, N. Jaber, M. Younis, and H. Fariborzi, "A Compact Adder and Reprogrammable Logic Gate Using Microelectromechanical Resonators with Partial Electrodes", IEEE Trans. Circuits Syst. II: Exp. Briefs, 2019. Available: 10.1109/tcsii.2019.2899938.

[9] D. N. Guerra, M. Imboden, and P. Mohanty, "Electrostatically actuated silicon-based nanomechanical switch at room temperature," Appl. Phys. Lett., vol. 93, no. 3, pp. 033515-33523, 2008.

[10]F. N. U. Juanda, W. Shu, J. S. Chang, "A 10GS/s 4bit single-core digital-to-analog converter for cognitive ultra-widebands", IEEE Trans. Circuits Syst. II Exp. Briefs, vol. 64, no. 1, pp. 16-20, 2017.

[11] L. Zhao, J. He, Y. Cheng, "A 6bit 4GS/s Currentsteering Digital-to-Analog Converter in 40nm CMOS with Adjustable Bias and DfT Block", International Conference on ASIC (ASICON), pp. 1-4, 2015.

\section{CONTACT}

*H. Fariborzi, hossein.fariborzi@kaust.edu.sa 